Intersections

Canadian Journal of Music

Revue canadienne de musique
Intersections CANADAN TOURAAL OP NUST

\title{
Sophie-Anne Leterrier. 2005. Le mélomane et l'historien. Paris : Armand Colin. 227 p. ISBN 2-200-26914-5 (couverture souple)
}

\section{Pascal Valois}

Volume 27, numéro 1, 2006

URI : https://id.erudit.org/iderudit/1013167ar

DOI : https://doi.org/10.7202/1013167ar

Aller au sommaire du numéro

\section{Éditeur(s)}

Canadian University Music Society / Société de musique des universités canadiennes

ISSN

1911-0146 (imprimé)

1918-512X (numérique)

Découvrir la revue

Citer ce compte rendu

Valois, P. (2006). Compte rendu de [Sophie-Anne Leterrier. 2005. Le mélomane et l'historien. Paris : Armand Colin. 227 p. ISBN 2-200-26914-5 (couverture souple)]. Intersections, 27(1), 120-125. https://doi.org/10.7202/1013167ar

All Rights Reserved (C) Canadian University Music Society / Société de musique des universités canadiennes, 2007
Ce document est protégé par la loi sur le droit d'auteur. L'utilisation des services d’Érudit (y compris la reproduction) est assujettie à sa politique d'utilisation que vous pouvez consulter en ligne.

https://apropos.erudit.org/fr/usagers/politique-dutilisation/ 
Riethmüller, Albrecht, et Hyesu Shin, dir. 2004. Busoni in Berlin : Facetten eines kosmopolitischen Komponisten. Stuttgart : Franz Steiner Verlag.

Roberge, Marc-André. 1991. Ferruccio Busoni : A Bio-Bibliography. Bio-

Bibliographies in Music, vol. 34. New York, Westport, Conn., et Londres : Greenwood Press.

1992. Recension de L'esthétique musicale de Ferruccio Busoni. Revue de musique des universités canadiennes, $\mathrm{n}^{\circ}$ 12/1 (1992) : 123-30. 269-305.

Weindel, Martina. 1996. Ferruccio Busonis Ästhetik in seinen Schriften und Briefen. Veröffentlichungen zur Musikforschung, vol. 18. Wilhelmshaven : Florian Noetzel.

MARC-ANDRÉ ROBERGE

Sophie-Anne Leterrier. 2005. Le mélomane et l'historien. Paris : Armand Colin. 227 p. ISBN 2-200-26914-5 (couverture souple).

Depuis le milieu du $\mathrm{XX}^{\mathrm{e}}$ siècle, les questionnements de type historiographique ont pris une place de plus en plus importante parmi les travaux des historiens. Ceux-ci ont en effet senti le besoin de remettre en cause les fondements de leur discipline. La musicologie, notamment en France, n’a pas échappé à cet autoexamen. Aux sources de cette investigation, on retrouve les musicologues français François Lesure, qui a écrit plusieurs articles sur le sujet, dont Les débuts de la musicologie française (1971), et Philippe Vendrix, qui a publié louvrage de référence Aux origines d'une discipline historique : La musique et son histoire en France aux XVII et XVIII e siècles (1993). Plus récemment, la question a été étudiée par Rémy Campos et Joël-Marie Fauquet, dont plusieurs articles se retrouvent dans le Dictionnaire de la musique en France au XIX $X^{e}$ siècle (2003) $)^{1}$. Le champ d'investigation était cependant en attente d'un ouvrage qui tracerait un portrait global de la situation.

Bien qu'envisagé dans une perspective beaucoup moins érudite, louvrage de Sophie-Anne Leterrier, Le mélomane et l'historien, se veut un peu le pendant, pour le XIX ${ }^{e}$ siècle, de la somme de Vendrix. L'auteure y brosse un panorama qui dépasse largement le simple portrait de la musicologie institutionnelle française. Il y est autant question de phénomènes comme la mode des opéras historiques ou les premiers concerts de musique ancienne ${ }^{2}$ que de la production d'ouvrages dérudition de type historiographique. Précisons que Leterrier, professeure d'histoire à l'Université d'Artois, est une spécialiste de l'histoire des disciplines scientifiques au $\mathrm{XIX}^{\mathrm{e}}$ siècle. On lui doit plusieurs publications sur le sujet, dont

1 Voir respectivement les articles " Histoire de la musique » et " Musicologie ".

2 Cet aspect a cependant été récemment traité en profondeur par la musicologue anglaise Katharine Ellis dans un ouvrage aussi exemplaire pour la forme que le fond : Interpreting the Musical Past : Early Music in Nineteenth-Century France (2005). 
L'Institution des sciences morales (1995) et Le XIX ${ }^{e}$ siècle historien (1997). Son travail s'inscrit dans une perspective interdisciplinaire.

Dans l'introduction, Leterrier décrit clairement l'objectif du livre : il s'agit de comprendre « comment et pourquoi l'esprit historique [...] s'est étendu au domaine musical » en France entre la Révolution et la Première Guerre mondiale (p. 14). Les 13 chapitres du livre se suivent de façon principalement chronologique et la plupart d’entre eux correspondent plus ou moins à une période de temps. Par exemple, le portrait de l'historien de la musique Alexandre-Étienne Choron (1771-1834) est partagé entre les troisième, quatrième et septième chapitres car son activité sétend sur plusieurs décennies. Lauteure utilise toutefois cette forme d'organisation avec souplesse. D'abord, certains chapitres sont construits de façon plus thématique que chronologique. C'est notamment le cas du neuvième chapitre, où l'auteure fait le point sur la musicologie à l'extérieur de la France. Ensuite, elle se permet souvent des bonds dans le temps lorsque la compréhension du discours l'exige. L'ouvrage possède également un deuxième niveau d'organisation. Il est en effet divisé en trois grandes parties intitulées "Troubadours et romantiques ", " Patrimoine et nations " et " Les voies de la démocratisation ". Ces trois axes détudes apparaissent comme une forme de leitmotiv à l'intérieur de chaque partie.

La méthodologie qui a présidé à lélaboration de louvrage comporte trois facettes. Premièrement, l'auteure a préféré traiter un nombre restreint de points plutôt que de procéder à un examen exhaustif. Par exemple, afin d'illustrer l'évolution des thèmes dans l'opéra historique, elle a choisi de se concentrer sur l'utilisation du personnage de Jeanne d'Arc, de la Révolution jusqu'au début du XXe siècle. Deuxièmement, elle a effectué une investigation minutieuse des sources primaires : archives du Conservatoire de Paris, divers documents soumis aux gouvernements (propositions, rapports, mémoires, études, considérations), arrêtés des gouvernements, procès verbaux des séances de diverses institutions, correspondances privées et publiques, discours d'inauguration, etc. Troisièmement, même si les grandes théories historiques sont abordées ${ }^{3}$, il ne faut pas s'attendre à trouver d’analyse épistémologique de la musicologie française du XIX ${ }^{\mathrm{e}}$ siècle. Au contraire, la parole est laissée aux acteurs eux-mêmes à chaque fois que cela est possible, et c'est là la principale force de l'ouvrage.

Leterrier nous explique elle-même les limites de sa recherche en précisant que son discours " repose sur des écrits théoriques et des publications érudites plus que sur des compositions ou des interprétations " et qu'il ne vise pas «à rendre compte de lévolution du langage musical " (p. 14). Elle fournit ensuite des justifications sur le choix de la France comme limite géographique de létude. Cette décision s'appuie, selon elle, sur l'accessibilité des sources, sur le rôle de pionnier de la France dans le domaine historique et sur la centralisation de l'activité artistique à Paris, ce qui facilite l'analyse et la comparaison des phénomènes.

Afin de répondre au "comment " et au " pourquoi " du lien entre l'histoire et la musique, lauteure axe son propos sur deux pôles. Le premier pôle

3 Comme le paradigme du génie qui représente son époque, supporté entre autres par Diderot, Schubart et Hoffmann (p. 28). 
(le " comment ") concerne la description des principales manifestations historico-musicales. D'abord, dans un siècle passionné pour l'histoire, l'érudition occupait une place de choix. Au centre des préoccupations des érudits de la musique se trouvait la remise en cause de la musique des Grecs comme objet détude quasi-exclusif. L'auteure démontre comment des musicographes comme Louis-François Perne (1772-1832) et Louis-François-Joseph Fétis (1784-1871) ont progressivement recentré le débat sur l'importance du Moyen Âge et de l'Église chrétienne dans l'histoire de la musique. Ce sont d’ailleurs les autorités cléricales, bien remises en selle après l'épisode révolutionnaire, qui ont entamé l'exhumation du plain-chant avec le concours des érudits Charles-Edmond de Coussemaker (1805-1876) et Normand Nisard (1812-1888). Ensuite, des institutions parisiennes ont aussi participé au goût de l'histoire. Parmi celles-ci, l'auteure analyse plus en profondeur la bibliothèque et le musée instrumental du Conservatoire de Paris, l'École de musique ancienne de Choron, l'École de musique sacrée de Niedermeyer et la Schola Cantorum de Paris. Enfin, Leterrier démontre comment l'esprit historique s'est étendu à deux champs principaux de l'activité musicale : l’opéra et le concert historique. La popularité du drame historique a traversé tout le XIX ${ }^{\mathrm{e}}$ siècle français, de Joseph de Méhul (1807), qui n'a d'historique que les personnages, en passant par Jeanne d'Arc de Gounod (1873), qui repose sur les travaux des historiens Wallon et Michelet, jusquà Pelléas et Mélisande de Debussy (1902), où le compositeur utilise l'histoire comme simple support à son imaginaire. Lauteure retrace aussi l'histoire du concert historique. Dès 1818, le violoniste et professeur au Conservatoire, Pierre Baillot (1771-1842), " est l'un des premiers à faire entendre à ses contemporains de la musique ancienne " (p. 73). Son exemple sera suivi avec plus de retentissement par Fétis, qui entraînera dans son sillage plusieurs cercles de musiciens amateurs. L'auteure, en habile généalogiste, observe que c'est chez un descendant de ces "clubs privés " de musique ancienne, le salon de Geneviève Thibaut de Chambure, que plusieurs vedettes actuelles de la musique ancienne (Christie, Savall, Kuijken, etc.) se rencontreront dans les années 1970 (p. 210).

Le deuxième pôle (le " pourquoi ») du livre concerne les motivations profondes du lien entre l'histoire et la musique. Premièrement, elle décrit comment le mouvement romantique, avec sa fascination pour le Moyen Âge et son goût pour le religieux, a favorisé la musique du passé. Dans ce contexte, les compositeurs dopéras et de chants multiplient les références au passé et le chant grégorien "est considéré comme un véritable "monument en péril" " (p. 69). Deuxièmement, les différents gouvernements qui se sont succédé au XIX ${ }^{\mathrm{e}}$ siècle ont encouragé la recherche historique en musique pour des raisons parfois complètement opposées. Leterrier nous donne quelques exemples : les révolutionnaires et les dirigeants de l'Empire ont cru voir une apologie de leurs régimes dans la société grecque antique et la reprise de ses idées dans l'opéra et dans la musique militaire; lors de la Restauration, les Bourbons (catholiques) ont donné une impulsion décisive aux recherches sur le chant grégorien; une large place a été faite à l'histoire sous la Monarchie de Juillet et la troisième République (dont le premier dirigeant, Adolphe Thiers, était lui-même historien). Troisièmement, lauteure nous montre à quel point, à partir des années 1820 jusqu'au $\mathrm{XX}^{\mathrm{e}}$ siècle, 
l'histoire est devenue un enjeu national en France. Elle cite l'entrée " Histoire " du Grand Dictionnaire universel du XIX ${ }^{e}$ siècle de Larousse, où Charles Monselet affirme que l'histoire " est destinée à devenir, au milieu de la civilisation moderne, ce que la théologie fut au Moyen Âge et dans l'Antiquité " (p. 200). Donc, pour elle, " [la musique] bénéficie de la promotion générale de l'histoire, de la valeur patrimoniale et nationale attachée aux œuvres anciennes" (p. 213).

Lintérêt de l'ouvrage réside d'abord dans l'approche documentaire étoffée qu'adopte Leterrier. Elle présente au lecteur une somme généreuse de documents dépoque qui situent avec nuance les protagonistes et leurs réalisations dans le contexte culturel, politique et religieux de leur temps. Le lecteur ne se sent cependant jamais submergé par les détails et le portrait global du sujet demeure toujours clair. Toutefois, le fait de couvrir un siècle entier tout en multipliant les références aux sources primaires exige des concessions. Lauteure a en effet dû exclure de sa démonstration certains musicographes importants comme Alexandre Vincent (1797-1868), François-Auguste Gevaert (18281908) et Joseph Louis d'Ortigue (1802-1866 ${ }^{4}$. On regrette aussi l'absence de Jérôme-Joseph de Momigny (1762-1842), d'Adrien de La Fage (1805-1862) et de Maurice Emmanuel (1862-1938), qui, selon Joël-Marie Fauquet, " figurent parmi les précurseurs authentiques de la musicologie moderne ». $(2003,834)$ Jean Gribenski (2006) nous rappelle enfin l'importance des médiévistes Pierre Aubry (1874-1910) et Jules Ecorcheville (1872-1915) dans la professionnalisation de l'activité de musicologue, tous deux absents de létude de Leterrier.

Leterrier adopte dans son ouvrage un style limpide et évite les écueils d'une écriture trop hermétique. De plus, il est presque impossible pour le lecteur de perdre le fil du propos. En effet, ce dernier est guidé à travers le livre par un généreux système hiérarchique comportant quatre niveaux : grandes parties, chapitres, sections de quelques pages et sous-sections de quelques paragraphes. Même si elles morcèlent quelque peu le discours, ces divisions clarifient la démonstration et orientent le lecteur. Enfin, malgré quelques défauts, l'appareil critique est étoffé et instructif.

Cependant, le livre Le mélomane et l'historien comporte plusieurs faiblesses techniques. D'abord, le lecteur doit se contenter d'un index des noms propres et, en l'absence d'une bibliographie, se rabattre sur les notes de bas de page s'il souhaite avoir un aperçu de la littérature. Les raisons qui ont justifié ces omissions sont difficiles à comprendre, surtout pour une étude de type " panoramique ». La tâche du chercheur qui veut utiliser le livre peut devenir carrément laborieuse. Ensuite, l'utilisation de la locution "op. cit. " dans les notes de bas de page rend souvent pénible la recherche des titres douvrages. Leterrier l'emploie même lorsqu'elle cite plusieurs ouvrages d'un même auteur et n'hésite pas à forcer le lecteur à revenir 60 pages en arrière s'il souhaite retrouver la réfé-

4 L'importance de ce dernier " musicien-littérateur » a récemment été mise en valeur par Sylvia L'Écuyer, qui a réuni une sélection importante de ses écrits dans ladmirable ouvrage : Joseph d'Ortigue, Écrits sur la musique, 1827-1846, textes réunis, présentés et annotés par Sylvia L'Écuyer (Paris : Société Française de Musicologie, 2003). 
rence complète 5 . Enfin, lauteure nomme systématiquement les personnages par leur nom de famille et ne fournit presque jamais leurs années de naissance et de mort. Elle devrait à tout le moins préciser les dates des principaux acteurs lorsqu'elle effectue des bonds géographiques ou chronologiques (ou les deux en même temps). Ce devrait être le cas pour Johann Gottfried Herder (1744-1803), qu'elle qualifie de "principal théoricien » de la musicologie allemande du XVIII siècle (p. 150).

Louvrage de Sophie-Anne Leterrier ne prétend pas à l'exhaustivité de travaux comme ceux d'Ellis (2005); l'auteur y brosse plutôt un tableau de la conscience historique en musique. Elle pose en ce sens des bases pour une réévaluation des acquis du XIX ${ }^{\mathrm{e}}$ siècle musicologique en France. Certains érudits, comme Fétis, ont en effet été victimes, dès la fin du XIX ${ }^{e}$, de la professionnalisation des musicologues et se sont vu apposer, pour la postérité, létiquette d'amateur, dans le pire sens du terme. Pourtant, Fétis fut l'un des premiers à remettre en cause la théorie du progrès dans les arts et à concrétiser sa position idéologique par ses concerts historiques. Enfin, si l'étude de Leterrier comblera les attentes d'un public cultivé, elle s'avérera moins satisfaisante pour les musicologues. Ceux-ci y trouveront néanmoins un bon panorama, qui pourra servir de point de départ à d'éventuelles recherches historiographiques.

\section{RÉFÉRENCES}

Campos, Rémy. 2003. "Histoire de la musique ". Dans Dictionnaire de la musique en France au XIX ${ }^{e}$ siècle, sous la direction de Joël-Marie Fauquet, 595. Paris : Fayard.

Ellis, Katharine. 2005. Interpreting the Musical Past : Early Music in NineteenthCentury France. Oxford et New York : Oxford University Press.

Fauquet, Joël-Marie, dir. 2003. Dictionnaire de la musique en France au XIX ${ }^{e}$ siècle. Paris : Fayard.

Fauquet, Joël-Marie. 2003. «Musicologie ». Dans Dictionnaire de la musique en France au XIX $X^{e}$ siècle, sous la direction de Joël-Marie Fauquet, 834. Paris : Fayard.

Gribenski, Jean. 2006. "Musicology, \$III : National traditions of musicology, France ", The New Grove Dictionary of Music Online, édité par Laura Macy [en ligne]. <http://www.grovemusic.com> (consulté le 3 juillet 2006).

Lesure, François. 1971. «Les débuts de la musicologie française ». Dans Memorie e contributi alla musica dal Medioevo all'età moderna offerti a Federico Ghisi nel settantesimo compleanno (1901-1971), Miscellanee saggi convegni, $2^{\mathrm{e}}$ vol. , 367-375. Bologne : A.M.I.S.

Leterrier, Sophie-Anne. 1995. L'Institution des sciences morales (1795-1850). Paris : L'Harmattan.

Leterrier, Sophie-Anne. 1997. Le XIX ${ }^{e}$ siècle historien: Anthologie raisonnée. Paris : Belin.

5 Néanmoins, les notes sont placées en bas de page (et non en fin de chapitre, ou, pire, à la fin du livre), ce qui facilite leur consultation. 
Ortigue, Joseph d'. 2003. Écrits sur la musique, 1827-1846. Textes réunis, présentés et annotés par Sylvia L'Écuyer. Paris : Société Française de Musicologie. Vendrix, Philippe. 1993. Aux origines d'une discipline historique: La Musique et son Histoire en France aux XVII et XVIII siècles. Bibliothèque de la Faculté de Philosophie et Lettres de l'Université de Liège, fascicule $n^{\circ} 260$. Genève : Librairie Droz.

Pascal Valois

Rupa Huq. Beyond Subculture. Pop, Youth and Identity in a Postcolonial World. London : Routledge, 2006. viii, 217 p. ISBN 0-415-27815-5 (couverture souple).

Ce livre de la sociologue britannique Rupa Huq, professeure à l'Université Kingston de Londres et spécialiste en youth culture, propose une mise à jour du phénomène des subcultures dans les études de la culture populaire (popular studies) ${ }^{1}$. Ces deux termes, youth culture et subculture, ont vu le jour dans les pays anglophones après la Deuxième Guerre mondiale. Le premier a été créé par le Chicago School of Urban Gang Sociology dans les années 1950, mais ce sont les travaux du Centre for Contemporary Cultural Studies (CCCS) de l'Université de Birmingham au Royaume-Uni qui lui donneront le sens que l'on connaît aujourd'hui'2.

Dès l'introduction, l'auteure indique la difficulté de définir ces termes clairement, surtout que ces définitions peuvent varier d'un chercheur à l'autre. En termes très généraux, les études en youth culture font référence aux phénomènes culturels par lesquels la jeunesse s'exprime, se distingue et se situe par rapport à la société dans laquelle elle vit. Le terme subculture, quant à lui, fait référence aux systèmes de valeurs, aux croyances, aux pratiques et conduites, aux normes par lesquelles un groupe de jeunes se définit face à l'ensemble de la société, face à lui-même et même vis-à-vis de ses adhérents. Ces normes véhiculent également une prise de position politique, culturelle ou sociale face à la société. Elles peuvent découler d'antécédents ethniques même si ces jeunes sont nés en Occident, ce que les diverses diasporas ont tendance à prouver.

Beyond Subculture est divisé en deux parties. La première donne un aperçu des fondements et développements théoriques des principales études en $s u b$ culture, surtout celles du CCCS de l'Université de Birmingham dans les années 1970, centre qui a été le fer de lance de ces études. Lauteure se livre à une critique détaillée de ces recherches qui, déjà vers la fin des années 1970, étaient sévèrement désapprouvées par plusieurs chercheurs, incluant même des membres

1 Comme les études de la culture populaire (popular studies) noont pas connu le même essor dans les pays de langue française que ceux de langue anglaise, la traduction française de certains termes génériques n’a pas toujours la même signification que dans la langue d’origine. Il mapparaît donc justifié ici de recourir aux termes anglais.

2 Les travaux menés par Stuart Hall/Tony Jefferson (1976) et Dick Hebdige (1979) sont parmi les plus connus. 